\title{
The comparability of the universalism value over time and across countries in the European Social Survey: exact vs. approximate measurement invariance
}

\author{
Florian Zercher $^{1 *}$, Peter Schmidt ${ }^{1}$, Jan Cieciuch ${ }^{2,3}$ and Eldad Davidov ${ }^{4}$ \\ ${ }^{1}$ Department of Political Science, University of Giessen, Giessen, Germany, ${ }^{2}$ University Research Priority Program "Social \\ Networks", University of Zürich, Zürich, Switzerland, ${ }^{3}$ Institute of Psychology, Cardinal Stefan Wyszyński University in \\ Warsaw, Warsaw, Poland, ${ }^{4}$ Institute of Sociology, University of Zürich, Zürich, Switzerland
}

\section{OPEN ACCESS}

Edited by:

Mike W.-L. Cheung,

National University of Singapore

Singapore

Reviewed by:

Suzanne Jak,

Utrecht University, Netherlands

Rens Van De Schoot

Utrecht University, Netherlands

*Correspondence:

Florian Zercher

Department of Political Science,

University of Giessen,

Karl-Glöcknerstrasse 21 E, 35394

Giessen, Germany

florian.zercher@sowi.uni-giessen.de

Specialty section:

This article was submitted to

Quantitative Psychology and

Measurement

a section of the journal

Frontiers in Psychology

Received: 11 October 2014

Accepted: 17 May 2015

Published: 04 June 2015

Citation:

Zercher F, Schmidt $P$, Cieciuch J and Davidov E (2015) The comparability of

the universalism value over time and across countries in the European Social Survey: exact vs. approximate

measurement invariance.

Front. Psychol. 6:733.

doi: 10.3389/fpsyg.2015.00733
Over the last decades, large international datasets such as the European Social Survey (ESS), the European Value Study (EVS) and the World Value Survey (WVS) have been collected to compare value means over multiple time points and across many countries. Yet analyzing comparative survey data requires the fulfillment of specific assumptions, i.e., that these values are comparable over time and across countries. Given the large number of groups that can be compared in repeated cross-national datasets, establishing measurement invariance has been, however, considered unrealistic. Indeed, studies which did assess it often failed to establish higher levels of invariance such as scalar invariance. In this paper we first introduce the newly developed approximate approach based on Bayesian structural equation modeling (BSEM) to assess cross-group invariance over countries and time points and contrast the findings with the results from the traditional exact measurement invariance test. BSEM examines whether measurement parameters are approximately (rather than exactly) invariant. We apply BSEM to a subset of items measuring the universalism value from the Portrait Values Questionnaire (PVQ) in the ESS. The invariance of this value is tested simultaneously across 15 ESS countries over six ESS rounds with 173,071 respondents and 90 groups in total. Whereas, the use of the traditional approach only legitimates the comparison of latent means of 37 groups, the Bayesian procedure allows the latent mean comparison of 73 groups. Thus, our empirical application demonstrates for the first time the BSEM test procedure on a particularly large set of groups.

Keywords: European Social Survey, approximate vs. exact measurement invariance, Portrait Value Questionnaire, universalism, Bayesian estimation, cross-national research, repeated cross-sections

Over the last decades, considerable research on values has taken place (Hitlin and Piliavin, 2004). These theoretical and empirical research contributions have been inspired especially by Inglehart and his colleagues (Inglehart, 1977; Inglehart and Welzel, 2005) and Schwartz and colleagues (Schwartz, 2003; Schwartz et al., 2012). Inglehart's value measurement instruments have been implemented in the World Value Survey (WVS), whereas a short version of Schwartz's Portrait Values Questionnaire (PVQ) with 21 items has been included in the European Social Survey (ESS). 
Comparisons of the two theoretical conceptions and the measurement instruments based on them were undertaken by Datler et al. (2013) and Beckers et al. (2012).

To date, the PVQ has been the object of extensive comparative research in the social sciences. Studies have focused, for example, on the relation between values and political behavior, leftright orientation, attitudes toward immigration, attitudes toward homosexuality and sociodemographic characteristics (Davidov et al., 2008, 2014b; Piurko et al., 2011; Meuleman et al., 2012; Schwartz et al., 2012; Kuntz et al., 2015) by making use of increasingly available cross-national data sources, such as the ESS or the WVS. The cross-national orientation in the study of values offered the advantage of introducing a stricter test of propositions (Popper, 2005), thereby expanding our knowledge about the validity of theories in different societies and allowing us to acquire insights into macro-micro effects (Opp, 2011). However, in comparative research, the issue of comparability across countries must be addressed (Davidov et al., 2014a). Respondents in different countries may understand survey questions in various ways (Latcheva, 2011; Braun et al., 2013) or respond in systematically different ways to the same questions (Harkness et al., 2010). This may lead to biased means, factor loadings and regression coefficients. Therefore, the assumption of cross-cultural measurement invariance needs to be tested (Meredith, 1993; Vandenberg and Lance, 2000; Davidov and Siegers, 2010; Millsap, 2011; Sarrasin et al., 2012; van de Schoot et al., 2012; Davidov et al., 2014a).

Davidov et al. (2008) and Davidov (2008, 2010) tested the measurement invariance properties of values across countries in three rounds of the ESS and could establish only metric invariance within the rounds across most countries and longitudinal scalar invariance within countries ${ }^{1}$. However, it remains to be answered if value measurements are invariant both across countries and over time and whether such an extensive test is feasible with real data. After all, various researchers who use values as explanatory or as explained constructs wish to test propositions referring simultaneously to different countries ("the cross-cultural aspect") and time points ("the dynamic aspect"). Such an endeavor requires that measurement invariance is given simultaneously over time and across countries. However, such a measurement invariance test has not been performed in the past. Moreover, such a test becomes increasingly important considering the continuous growth in the number of countries and time points in the large data-generating programs mentioned before. Thus, our research question is whether it is feasible to test and establish measurement invariance across a very large number of groups.

In the current study we would like to focus on the universalism value because it is the only value which was measured in the PVQ-21 with three (rather than only two) items, thus allowing us to control for all forms of random and nonrandom measurement errors (Bollen, 1989). Furthermore, this universalism scale has also been used in a considerable number of empirical studies using ESS data (Jowell et al., 2007; Beierlein et al., 2012; Davidov

${ }^{1}$ For an invariance test of a new scale to measure human values, see Cieciuch et al. (2014a,b). et al., 2012; Saris et al., 2013) and other datasets (Schwartz et al., 2012; van de Schoot et al., 2012). We will examine its simultaneous comparability across 15 countries and six time points using the new procedure for assessing approximate invariance using Bayesian estimation (van de Schoot et al., 2013). To the best of our knowledge, no previous study has assessed invariance across so many groups simultaneously ${ }^{2}$. We will demonstrate the application of the two approaches on the same large set of time/country groups. Given previous findings, we expect to find metric invariance at best for the universalism scale but no scalar invariance across countries using the traditional exact method. However, we expect to establish scalar invariance at least for a subset of countries using the approximate approach.

We begin by briefly presenting the traditional exact approach and then describe the new approximate approach to test for measurement invariance across groups. Next, we describe our data and the three items that measure universalism. In the empirical part we report the results of the two approaches to test for invariance. We finalize with a discussion of the pros and cons of the traditional exact approach vs. the approximate approach to test for measurement invariance in cross-national research.

\section{The Traditional Approach to Measurement Invariance Testing: Multi-Group Confirmatory Factor Analysis (MGCFA)}

Multi-group confirmatory factor analysis (Jöreskog, 1971; Bollen, 1989; Brown, 2006) has been the most common method used to test for measurement invariance. There are three distinct and hierarchically ordered levels of measurement invariance. Each level is defined by the parameters constrained to be equal across groups. The first and lowest level is configural invariance (Horn and McArdle, 1992; Meredith, 1993; Vandenberg and Lance, 2000). Configural invariance requires that each construct is measured by the same items. The second level is metric invariance, and it guarantees that the measured construct essentially has the same meaning in the different groups under study. Full metric invariance is tested by constraining the factor loadings to be equal across the groups to be compared (Vandenberg and Lance, 2000). If full metric invariance is established, a one-unit increase in the latent construct has the same meaning across groups. Subsequently, covariances and unstandardized regression coefficients may be meaningfully compared across samples (Steenkamp and Baumgartner, 1998). However, it is still uncertain whether the construct is measured on the same scale (Horn and McArdle, 1992; Steenkamp and Baumgartner, 1998; Vandenberg and Lance, 2000). Scalar invariance requires, in addition, that the intercepts are equal across groups. It is tested by constraining both the factor loadings and the intercepts to be equal across the groups to be compared (Vandenberg and Lance, 2000). If full scalar invariance

\footnotetext{
${ }^{2}$ In the study of van de Schoot et al. (2013), only a small number of groups was studied. Cieciuch et al.'s (2014a) studies contained eight groups, and Davidov et al. (2015) contained 15 groups in six separate tests. None of these studies performed a simultaneous test over countries and time points, which would have led to a much higher number of groups.
} 
is established, also the means may be meaningfully compared across groups (Steenkamp and Baumgartner, 1998).

Below, the corresponding three sets of constraints for the three levels of invariance are defined for a particular item in a one-factor case for individual $i$ in group $j$ (see Muthén and Asparouhov, 2013).

$$
\begin{aligned}
\text { Configural invariance } y_{i j} & =v_{j}+\lambda_{j} f_{i j}+\varepsilon_{i j} \\
E\left(f_{i}\right) & =\alpha_{j}, V\left(f_{j}\right)=\psi_{j}
\end{aligned}
$$

Where $\mathrm{v}$ is a measurement intercept, $\lambda$ is a factor loading, $\mathrm{f}$ is a factor with mean $\alpha$ and variance $\Psi$, and $\varepsilon$ is a residual with mean zero and variance $\theta$, uncorrelated with $f$. The configural model has subscript $j$ for both intercepts and loadings.

$$
\begin{aligned}
\text { Metric invariance } y_{i j} & =v_{j}+\lambda f_{i j}+\varepsilon_{i j} \\
E\left(f_{i}\right) & =\alpha_{j}=0, V\left(f_{j}\right)=\psi_{j}
\end{aligned}
$$

The metric model drops the subscript $j$ for the loadings because they are assumed to be equal.

$$
\begin{aligned}
\text { Scalar invariance } y_{i j} & =v+\lambda f_{i j}+\varepsilon_{i j} \\
E\left(f_{i}\right) & =\alpha_{j}, V\left(f_{j}\right)=\psi_{j}
\end{aligned}
$$

The scalar model drops the subscript $j$ for both intercepts and loadings because they are assumed to be equal ${ }^{3}$.

In practice, it is particularly difficult to reach full scalar invariance. Variations in the way respondents react to questions or systematic response biases such as social desirability or acquiescence (Billiet et al., 2003; Oberski et al., 2012), which may be individually or culturally determined, could possibly distort responses to the extent that scalar invariance will not exist in most empirical applications (Davidov et al., 2014a). There have been basically two major approaches to handling the issue of measurement noninvariance (Jouha and Moustaki, 2013; van de Schoot et al., 2013; Davidov et al., 2014a):

(1) Ignoring it. This is what the overwhelming majority of researchers have done as is evident in publications using cross-national and multigroup data, repeated cross-sections and panel data (see Davidov et al., 2014a). This line of literature has typically used sum scores instead of first testing whether the assumption of invariance can be supported by the data. As Steinmetz (2013) demonstrated in a Monte Carlo study, the use of sum scores is not an adequate procedure without invariance testing, as sum score differences are only warranted in conditions of full measurement invariance.

(2) Byrne et al. (1989) and Steenkamp and Baumgartner (1998) proposed the concept of partial invariance as a sufficient condition for meaningful cross-group comparisons. This approach has become a standard approach among various researchers. Partial invariance is given if the parameters of at least two indicators per construct (i.e., loadings for partial metric invariance and loadings plus intercepts for partial scalar invariance) are equal across groups.

${ }^{3}$ In the Analytical Strategy section we shortly describe our approach to identify the models.
Several scholars rely on partial invariance when comparing countries, cultures or other units of analysis. However, even partial scalar invariance may often be rejected.

Three common procedures in the MGCFA literature which rely on global fit measures have been proposed to evaluate whether measurement invariance is established:

(1) To rely on the chi-square difference test and compare the configural, metric and scalar invariance models, which form nested models (Jöreskog, 1978; Bollen, 1989; Meredith, 1993; Brown, 2006). According to this procedure, the chi-square difference test is used to assess the correctness of the model. However, the use of the chi-square difference test has been criticized because of its sensitivity to sample size (among other reasons) (Jöreskog, 1993; Cheung and Rensvold, 2002).

(2) To use cut-off values for the difference in the comparative fit index (CFI), the root mean square error of approximation (RMSEA) and the standardized root mean square residual (SRMR) (Chen, 2007; for alternative cut-off values see Meade et al., 2008). According to this procedure, if the change in model fit is smaller than the criteria proposed in the literature, measurement invariance for that level is established. According to the results of Chen's (2007) simulation study, the following recommendations have been proposed:

(a) If the sample size is larger than 300 , metric noninvariance is indicated by a change in CFI larger than 0.01 supplemented by a change in the RMSEA larger than 0.015 or a change in SRMR larger than 0.03 compared with the configural invariance model.

(b) Scalar noninvariance is evidenced by a change in CFI larger than 0.01 supplemented by a change in RMSEA larger than 0.015 or a change in SRMR larger than 0.01 compared with the metric invariance model.

(3) The third procedure suggests employing the Akaike information criterion (AIC) and the Bayesian information criterion (BIC) information theoretic measures to compare the configural, metric and scalar invariance models (Kass and Raftery, 1995). Following the criteria proposed by Kass and Raftery (1995), a very strong difference is indicated when the AIC or BIC difference is greater than $1^{4}$.

Since empirical tests often fail to establish measurement invariance based on these criteria, it has been argued that the criteria for testing measurement invariance may be too strict (Muthén and Asparouhov, 2013) and that more liberal criteria should be used to assess approximate (rather than exact) measurement invariance.

\footnotetext{
${ }^{4} \mathrm{~A}$ more detailed analysis of the issue of robustness against violations of metric and scalar invariance is given in Jouha and Moustaki (2013), Oberski (2014), and Meuleman (2012). See also Saris et al. (2009) for an alternative procedure to assess whether exact measurement invariance is given which relies on identifying local misspecifications while taking the power of the test into account. Furthermore, Thompson and Green (2013) argue that it might be better to rely on theory and past empirical findings and to be less dependent on empirical methods like the global fit measures and the modification indices when deciding whether to accept or reject a given level of invariance. This issue has not been settled yet.
} 


\section{The Bayesian Approach to Test for Approximate Measurement Invariance}

Recently, Muthén and Asparouhov (2013) and van de Schoot et al. (2013) proposed an alternative approach to test for measurement invariance by applying approximate Bayesian measurement invariance testing. The exact procedure, which constrains factor loadings and intercepts to be exactly equal to establish measurement invariance, is very restrictive and rarely establishes invariance (Jouha and Moustaki, 2013; van de Schoot et al., 2013). Approximate measurement invariance permits "small" differences between parameters (van de Schoot et al., 2013). The parameters specified in a Bayesian approach are considered to be variables, and their distribution is described by priors. The assignment of prior distributions to unknown parameters reflects the researcher's uncertainty about them regardless of whether one conceives of a parameter as having one true value or not (Levy and Choi, 2013). Such uncertainty may be applied for various parameters both in single-group CFA and MGCFA. In invariance testing one may assume that differences between parameters (factor loadings, intercepts) are approximately equal. Thus, we would allow the introduction of some uncertainty by specifying a small variance of, for example, 0.01 or 0.05 around the difference in factor loadings or intercepts (van de Schoot et al., 2013).

Figure 1 delineates the difference between the traditional exact approach to test for measurement invariance and the Bayesian approximate approach. In the traditional exact approach, the differences of factor loadings $(\lambda)$ or intercepts $(v)$ between groups are assumed to be exactly zero, while in the Bayesian approach the differences are assumed to be approximately zero with a mean of zero and some small variance delta $(\delta)$. Thus, we allow small variations in a given interval between the parameters as part of the measurement model ${ }^{5}$ (see also Kruschke et al., 2012; Muthén and Asparouhov, 2012, 2013; Levy and Choi, 2013). Simulations suggest that "small" variations may be allowed without risking invalid conclusions in comparative research (van de Schoot et al., 2013).

The difference between the traditional exact approach and the Bayesian approximate approach is also evident in the

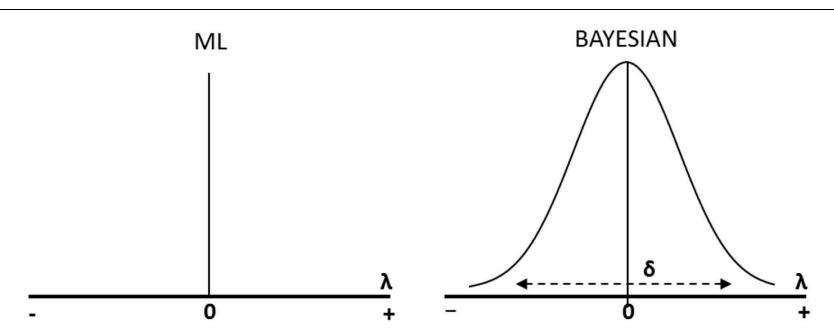

FIGURE 1 | Difference in parameter estimation between Maximum Likelihood (ML) and the Bayesian approach (see Muthén and Asparouhov, 2013; van de Schoot et al., 2013).

\footnotetext{
${ }^{5}$ Whether and to what extent our analysis procedure corresponds with the common concept of using prior knowledge in the same way as in Bayesian statistics is debatable, since our priors actually correspond to an assumption testing of approximate invariance rather than strictly to prior knowledge.
}

definitions of the confidence interval (used in the traditional exact approach) and the credibility interval (CI) (used in the Bayesian approximate approach). The confidence interval over an infinite number of samples taken from the population expresses that $95 \%$ of these contain the true population value. By way of contrast, the CI expresses that there is a $95 \%$ probability that the population value is within the limits of the interval.

A number of fit measures have been proposed to specifically assess Bayesian models (Gelman, 2003, 2013; Levy, 2011). These fit measures can detect if the actual deviations are larger than those allowed by the researcher in the prior distribution. First, the model fit can be evaluated based on the posterior predictive probability value ( $\mathrm{ppp}$ ). The ppp is computed by comparing two types of information: the discrepancy between the model and the observed data and the discrepancy between the model and the posterior predicted data (Levy and Choi, 2013, p. 597) ${ }^{6}$. According to Muthén and Asparouhov (2012) and van de Schoot et al. (2013), the ppp value of a model that fits the data should be nonsignificant, and if it is around 0.50 , it indicates a well-fitting model.

A second fit measure refers to the $\mathrm{CI}$ for the difference between the observed and the replicated chi-square values. According to Muthén and Asparouhov (2012) and van de Schoot et al. (2013), the CI should contain zero. Finally, the BIC (Schwarz, 1978) and the deviance information criterion (DIC) (Spiegelhalter et al., 2002) were also proposed for the assessment of model comparison in a Bayesian framework (Kass and Raftery, 1995). $\mathrm{BIC}$ is computed using the following formula:

$$
B I C=-2 \ell(\hat{\theta} \mid X)+p * \ln (n)
$$

where $\ell(\hat{\theta} \mid X)$ is the maximized log-likelihood, $p$ is the number of parameters, and $n$ is the number of observations. Building on this tradition of comparing values of information criteria, Spiegelhalter et al. (2002) introduced the DIC:

$$
D I C=\overline{D(\theta)}+p_{D}=2 \overline{D(\theta)}-D \overline{(\theta)}+2 p_{D}
$$

where $D(\theta)$ is the posterior mean of the deviation (negative of twice the log-likelihood function), $p_{D}$ is a complexity measure defined as the difference between the posterior mean of the deviance and the deviance evaluated at the posterior mean, $\overline{D(\theta)}$.

Testing for approximate measurement invariance consists of two steps. The first identifies the noninvariant parameters while fitting the model to data. Noninvariant parameters are those parameters which are found to be outside of the "wiggle room" allowed for the parameter differences. In the second step the noninvariant parameters are freed and the model is recalculated (Muthén and Asparouhov, 2013; van de Schoot et al., 2013). In the next section we are going to provide a practical application by demonstrating a test for approximate invariance using ESS data.

\footnotetext{
${ }^{6}$ This procedure corresponds to the comparison between the observed variancecovariance matrix $(S)$ and the expected variance-covariance matrix $\left(\sum\right)$ using maximum likelihood estimation in structural equation modeling (Bollen, 1989). ${ }^{7}$ For a discussion of other fit measures for Bayesian SEM, see Kaplan (2014) and Levy and Choi (2013). Indeed, as Levy and Choi(2013, p. 599) argue, little research has been conducted on the relative merits and limitations of these fit measures to evaluate model comparisons in BSEM.
} 
TABLE 1 | ESS sample sizes for the selected 15 countries over six ESS rounds (2002-2012).

\begin{tabular}{|c|c|c|c|c|c|c|c|}
\hline & $\begin{array}{c}\text { 1st Round } \\
(2002 / 3)\end{array}$ & $\begin{array}{c}\text { 2nd Round } \\
\text { (2004/5) }\end{array}$ & $\begin{array}{c}\text { 3rd Round } \\
(2006 / 7)\end{array}$ & $\begin{array}{l}\text { 4th Round } \\
\text { (2008/9) }\end{array}$ & $\begin{array}{c}\text { 5th Round } \\
(2010 / 11)\end{array}$ & $\begin{array}{l}\text { 6th Round } \\
(2012 / 13)\end{array}$ & $N$ \\
\hline Belgium & 1899 & 1778 & 1798 & 1760 & 1704 & 1869 & 10,808 \\
\hline Germany & 2919 & 2870 & 2916 & 2751 & 3031 & 2958 & 17,445 \\
\hline Denmark & 1506 & 1487 & 1505 & 1610 & 1576 & 1650 & 9334 \\
\hline United Kingdom & 2052 & 1897 & 2394 & 2352 & 2422 & 2286 & 13,403 \\
\hline Hungary & 1685 & 1498 & 1518 & 1544 & 1561 & 2014 & 9820 \\
\hline Ireland & 2046 & 2286 & 1800 & 1764 & 2576 & 2628 & 13,100 \\
\hline Netherlands & 2364 & 1881 & 1889 & 1778 & 1829 & 1845 & 11,586 \\
\hline Norway & 2036 & 1760 & 1750 & 1549 & 1548 & 1624 & 10,267 \\
\hline Slovenia & 1519 & 1442 & 1476 & 1286 & 1403 & 1257 & 8383 \\
\hline N & 29,415 & 28,441 & 28,492 & 28,800 & 28,317 & 29,606 & 173,071 \\
\hline
\end{tabular}

\section{Method and Data}

For the analysis we employ data from the ESS measuring the universalism value (Schwartz, 2003; Schwartz et al., 2012) ${ }^{8}$. The ESS is a biannual cross-national European survey that is administered to representative samples from approximately 30 countries. Since its inception in 2002/2003, it has included questions that measure values in its core module. These questions have been repeated in each round and used extensively in crossnational research. In the present analysis we have included 15 countries which participated in all six rounds. Table 1 presents the sample sizes for each country/time point combination between 2002 and 2012 .

Three items were used to measure the universalism value. Respondents were presented with a descriptive portrait of a person (gender matched), and they were requested to indicate to what extent they were similar to this person. The response scale ranged from 1 (very much like me) to 6 (not like me at all). These responses were reversed so that higher scores represented greater similarity to enable a more straightforward interpretation of the scores. The correlations between items were considerable and ranged approximately between 0.3 and 0.4 . The rate of missing values for these items ranged from 4.0 to $4.2 \%$ only for each country/time point combination. Table 2 presents the item formulations.

\section{Analytical Strategy}

\section{Testing for Exact (Full or Partial) Invariance}

In the first step we performed six MGCFAs (one for each round) across 15 countries, and after that, the analysis was performed on all 15 countries and six rounds (with a total of 90 groups)

${ }^{8}$ The raw data is available at the official site of the European Social Survey: http:// www.europeansocialsurvey.org/downloadwizard.
TABLE 2 | Formulation of universalism items.

"Now I will briefly describe some people. Please listen to each description and tell me how much each person is or is not like you. Use this card for your answer..."

Universalism Item1-“...She/he thinks it is important that every person in the world should be treated equally. She/he believes everyone should have equal opportunities in life."

Universalism Item2-“...It is important to her/him to listen to people who are different from her/him. Even when she/he disagrees with them, she/he still wants to understand them."

Universalism Item3-“...She/he strongly believes that people should care for nature. Looking after the environment is important to her/him."

simultaneously. In both cases, the full information maximum likelihood (FIML) procedure was used to deal efficiently with the problem of missing values (Schafer and Graham, 2002). We used the robustified maximum likelihood estimation procedure to deal with the ordered categorical character of the data ${ }^{9}$.

Each analysis contained assessments for configural, metric and scalar invariance, with the corresponding constraints for each level of the measurement invariance ${ }^{10}$. In a second step, when full measurement invariance was not established, we tried to assess partial measurement invariance. In order to establish partial scalar invariance (where at least two items are constrained to be exactly equal), the intercept of only one item was released, because partial scalar invariance requires that parameters of at least two items are constrained to be equal across all groups.

${ }^{9}$ Only standard errors and chi-square differ between MLR and FIML.

${ }^{10}$ To identify the model we used the marker variable method (MVM; see Little et al., 2006). We constrained the factor loading of one item to one and its intercept to zero. To test the robustness of our findings, we re-ran the model two more times, and each time with a different item as the marker item. The results remained essentially the same. 


\section{Testing for Approximate Invariance}

Following Muthén and Asparouhov (2013) and van de Schoot et al. (2013), we ran models with informative priors with a mean of zero and variances of $0.005,0.01,0.05$, and 0.5 for the differences between factor loadings or intercepts across groups ${ }^{11}$. Next, we identified in each model with the different priors those factor loadings and intercepts which were different. In the next step we freed all parameters which were considerably different across groups and left the informative priors for all the other equality parameters intact (Muthén and Asparouhov, 2013). Table 3 summarizes the steps undertaken in each approach. These analyses were conducted on all ESS rounds and countries simultaneously.

\section{Results}

\section{The Traditional Exact Approach}

Table 4 presents the global fit measures of the accepted models after dropping countries using the traditional exact approach. The first part of the table presents the global fit measures of the accepted model in each round separately. The last part of the table presents the global fit measures for the accepted model in the simultaneous analysis across countries and rounds. After releasing the equality constraint on the intercept that had the highest modification index in most country/time point combinations (Byrne et al., 1989), we identified in the simultaneous analysis 53 country/time point combinations in

TABLE 3 | Analytical steps for the exact and the approximate measurement invariance approaches.

\begin{tabular}{lll}
\hline Traditional exact & Approximate approach \\
approach & & \\
\hline Steps & $\begin{array}{l}\text { 1. Configural model } \\
\text { 2. Metric model } \\
\text { 3. Scalar model }\end{array}$ & $\begin{array}{l}\text { 1. Setting different } \\
\text { informative priors for the } \\
\text { 4. Partial scalar model }\end{array}$ \\
$\begin{array}{l}\text { cross-group differences of } \\
\text { loadings and intercepts } \\
\text { 2. Releasing (approximate) } \\
\text { equality constraints (of } \\
\text { loadings and intercepts) that } \\
\text { are not supported by the } \\
\text { data }\end{array}$
\end{tabular}

$\begin{array}{lll}\text { Additional steps }^{12} & \begin{array}{l}\text { 5. Deleting groups which } \\ \text { are not fully or partially }\end{array} & \begin{array}{l}\text { 3. Deleting groups which } \\ \text { are not fully or partially }\end{array} \\ & \text { scalar invariant } & \begin{array}{l}\text { approximately invariant } \\ \text { and }\end{array}\end{array}$

As metric invariance could be established in the exact approach, we did not need to fall back to partial metric invariance.

\footnotetext{
${ }^{11}$ When running the Bayesian procedure, we first ran a model where the difference between factor loadings or intercepts across groups has a normal distribution prior with a mean of 0 and a very large variance of $10^{10}$ (the so-called noninformative prior). This allows us to firstly detect whether there are any calculation problems in the Bayesian analysis (van de Schoot et al., 2013).

${ }^{12}$ After we were unable to achieve partial measurement invariance using the common ways of model fitting, we had to delete countries/time points (groups) based on the modification indices for the exact approach and based on the single group ppp for the approximate approach.
}

which at least two items were noninvariant. These country/time point combinations had to be dropped from further analysis because, for these units, even partial invariance could not be established. For example, the items which measured the importance to understand different people and to take care of the environment were scalar noninvariant in Switzerland and Denmark at all measurement time points. Consequently, we dropped these countries from further analysis. Thus, in total, 37 of the country/time point combinations displayed partial exact scalar invariance ${ }^{13}$.

Furthermore, we employed AIC and BIC comparisons of the metric invariance and partial scalar invariance models (see Table 5) in the separate analyses for each round and in the simultaneous analysis. Following the criteria proposed by Kass and Raftery (1995) to compare BIC differences, we can conclude that all differences between the metric and the partial scalar model, in a reduced number of countries, are very large.

The results have two important implications. On the one hand, findings of partial scalar invariance allow meaningful mean comparison across 37 country/time point combinations for the universalism construct. However, it is discouraging to find out that mean comparisons of the universalism value may be problematic in so many of the country/time point combinations. Next, we turn to the approximate invariance test.

TABLE 4 | Global fit measures of the traditional exact approach.

\begin{tabular}{|c|c|c|c|c|c|}
\hline & $\mathrm{Chi}^{2}(\mathrm{df})$ & RMSEA & SRMR & CFI & $\begin{array}{c}\text { Countries/ } \\
\text { Timepoints }^{14}\end{array}$ \\
\hline \multicolumn{6}{|l|}{ ROUND 1} \\
\hline Partial scalar & 64.89 (24) & 0.029 & 0.029 & 0.985 & 8 \\
\hline \multicolumn{6}{|l|}{ ROUND 2} \\
\hline Partial scalar & 53.28 (28) & 0.022 & 0.027 & 0.992 & 9 \\
\hline \multicolumn{6}{|l|}{ ROUND 3} \\
\hline Partial scalar & $53.78(27)$ & 0.024 & 0.033 & 0.988 & 8 \\
\hline \multicolumn{6}{|l|}{ ROUND 4} \\
\hline Partial scalar & $87.43(24)$ & 0.040 & 0.041 & 0.978 & 8 \\
\hline \multicolumn{6}{|l|}{ ROUND 5} \\
\hline Partial scalar & $90.10(21)$ & 0.044 & 0.039 & 0.972 & 7 \\
\hline \multicolumn{6}{|l|}{ ROUND 6} \\
\hline Partial scalar & $69.26(21)$ & 0.034 & 0.036 & 0.980 & 7 \\
\hline \multicolumn{6}{|c|}{ COUNTRIES AND ROUNDS SIMULTANEOUSLY } \\
\hline Partial scalar & 348.23 (126) & 0.031 & 0.035 & 0.983 & $37^{15}$ \\
\hline
\end{tabular}

RMSEA, root mean square error of approximation; SRMR, standardized root mean square residual; CFI, comparative fit index; the partial scalar model corresponds to step 5 in Table 3.

\footnotetext{
${ }^{13}$ Discussing possible explanations why specific countries are not comparable to others is beyond the scope of the present study. See Davidov et al. (2012) for using multilevel structural equation modeling for explaining noninvariance.

${ }^{14}$ For the single rounds this refers to countries; for all rounds this is combination of countries and time points.

${ }^{15}$ Countries still included are: Belgium 2002-2012; Spain 2002-2006; Finland 2006-2010; United Kingdom 2012; Hungary 2002-2008; Ireland 2008, 2010; Netherlands 2002-2012; Norway 2004-2012; Poland 2006; Portugal 2004-2008; Sweden 2012; Slovenia 2002, 2006.
} 
TABLE 5 | AIC and BIC fit measures of the traditional exact approach ${ }^{16}$.

\begin{tabular}{llrr}
\hline & & AIC & \multicolumn{1}{c}{ BIC } \\
\hline Round 1 & Metric & 232453.884 & 2333335.682 \\
& Partial scalar & 133004.879 & 133373.601 \\
Round 2 & Metric & 218452.710 & 219328.143 \\
& Partial scalar & 134813.330 & 135221.803 \\
Round 3 & Metric & 222284.379 & 223163.765 \\
& Partial scalar & 106349.111 & 106687.021 \\
Round 4 & Metric & 225469.593 & 226350.568 \\
& Partial scalar & 109976.943 & 110337.466 \\
Round 5 & Metric & 226639.903 & 227520.419 \\
& Partial scalar & 98034.755 & 98344.903 \\
Round 6 & Metric & 237036.130 & 237923.153 \\
& Partial scalar & 113273.097 & 113589.931 \\
All rounds & Metric & 1362329.608 & 1368665.132 \\
& Partial scalar & 537676.482 & 539559.803 \\
\hline
\end{tabular}

\section{The Bayesian Approximate Approach}

Here, too, we first tested each round separately and then all rounds simultaneously ${ }^{17}$. Approximate measurement invariance across all countries was established in only two rounds (2002 and 2004). Next, as recommended by van de Schoot et al. (2013), we ran the model that included all time points and countries, using several prior variances to compare them. We released equality constraints on those loadings and intercepts which were different ${ }^{18}$. Finally, we deleted groups which were not approximately invariant. Table 6 reports the results for the model with a prior of 0.05 (Muthén and Asparouhov, 2013; van de Schoot et al., 2013).

Accordingly, 73 countries/time points remained in the model. Thus, the results suggest that the exact and approximate measurement invariance approaches produce quite different findings. Whereas, partial approximate scalar measurement invariance was established in 73 ESS country/time point combinations, exact scalar measurement invariance was only established in 37 country/time point combinations. In other words, the approximate test allows us to perform mean comparisons of universalism across a very large set of countries and time points.

\section{Mean Comparison}

We compared the country means obtained from the MGCFA and Bayesian analyses with each other as well as with those based on the raw sum scores for the 73 comparable country/time point combinations. This was done by estimating mean scores based on the exact and approximate approaches and comparing them

\footnotetext{
${ }^{16}$ The partial scalar model corresponds to step 5 in Table 3.

${ }^{17}$ An example of the syntax can be found in the Supplementary Material. We would like to thank Bengt and Linda Muthén very much for making it possible to run such a model in the Mplus 7.3 version (Muthén and Muthén, 1998-2014). Previous versions did not allow the inclusion of this number of groups.

${ }^{18} \mathrm{~A}$ detailed report of the results is beyond the scope of the present study and may be provided by the first author upon request.
}

TABLE 6 | Global fit measures for the approximate invariance test (mean = 0 and variance $=0.05$ ).

\begin{tabular}{lccc}
\hline ppp & $\begin{array}{c}\text { ppp after releasing } \\
\text { misspecified parameters }\end{array}$ & $\begin{array}{c}\text { Cl after releasing } \\
\text { misspecified parameters }\end{array}$ \\
\hline 90 groups $^{19}$ & 0.000 & 0.000 & $125.830-346.761$ \\
73 groups $^{19}$ & 0.026 & 0.052 & $-10.834-171.115$
\end{tabular}

ppp, posterior predictive probability; Cl, credibility interval.

TABLE 7 | Correlations between latent means computed using sum scores (1), the exact (2) and the approximate (3) measurement invariance models for 73 county/time points.

\begin{tabular}{cccc}
\hline & Sum scores (1) & Exact test $^{20}$ (2) & Approximate Bayesian test (3) \\
\hline 1 & 1 & & \\
2 & $0.997^{\star \star}$ & 1 & 1 \\
3 & $0.851^{\star \star}$ & $0.844^{\star \star}$ & 1
\end{tabular}

${ }^{* *} p<0.01$ (pairwise deletion).

to each other and to those computed using the raw data. Finally, we estimated the correlation between the means computed in the country/time point combinations based on each of the three procedures.

As Table 7 demonstrates, the correlation is highest between sum scores and the exact test (0.997), and the correlation between the Bayesian approximate test and the exact test (0.844) is lowest. Since the latent means from the approximate test are the only ones which rely on an acceptable model fit, we conclude that latent means based on the other approaches (the exact and the sum scores) are biased. Figure 2 presents the differences in the means between the sum scores and the scores from the approximate approach on a scatter plot. If the scores in the two methods were equal, they would all be on the diagonal. Stated another way, increased distance from the diagonal indicates increased differences between the scores.

Conclusions may also be biased when sum scores are compared for the same country longitudinally. Figure 3 presents the mean over time and within countries. For example, as Figure 3 demonstrates, when comparing the sum scores in Poland, one would assume that the means considerably increased between 2002 and 2012. However, based on the approximate approach, the data show that there was no mean difference between 2002 and 2012 for the universalism value scores in Poland. By way of contrast, the sum scores indicate no mean difference between 2002 and 2012 in Ireland. However, according to the approximate test, there was a slight increase in the universalism mean in Ireland between the two rounds. We thus conclude that if a researcher would draw conclusions based on the composite scores, either to compare countries with each other

\footnotetext{
${ }^{19}$ Countries/time points not included are Denmark 2002, 2004, 2010, 2012; Spain 2008, 2010, 2012; Finland 2002, 2004; United Kingdom 2010; Hungary 2008; Ireland 2012; Norway 2008; Poland 2008, 2010; Sweden 2012; Slovenia 2010.

${ }^{20}$ To illustrate the comparison, these latent means are based on the model with all countries from the exact test that did not achieve scalar invariance.
} 


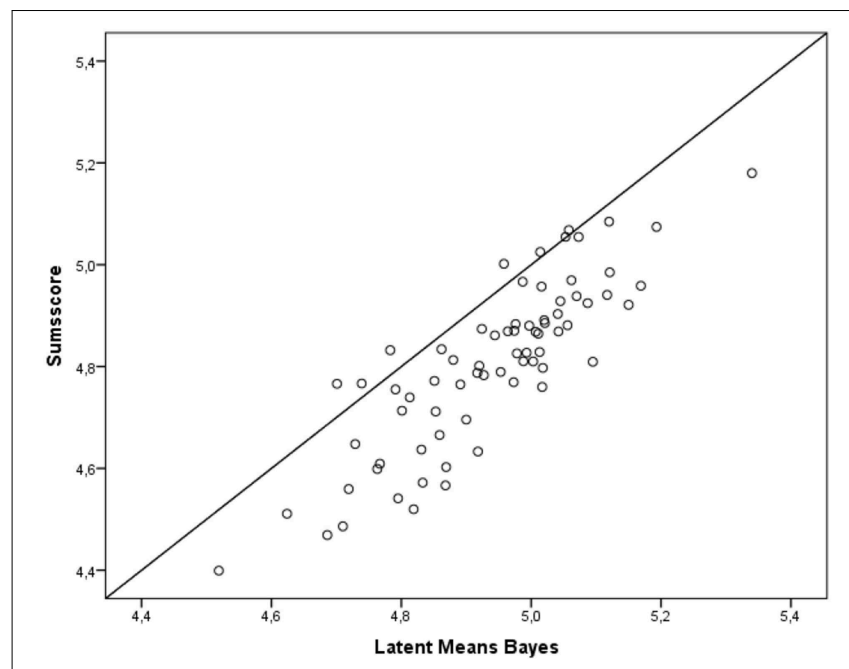

FIGURE 2 | Relationship between sum scores and scores based on the Bayesian estimation in 73 country/time point combinations.

or to compare scores within the same country and over time, they might be misled by the scores and reach wrong conclusions. In Figure 3 one can see the variance of the latent means over the six time points. The length of the line shows the variation and the colored circles show the latent mean of universalism at in each round.

Finally, Figure 4 displays the mean development of universalism over time in each of 15 countries and how this compares to the overall mean level of universalism across all countries and rounds.

To visualize the latent means over different time points and different countries, we split the countries into three groups comprising five countries each. The straight, dotted horizontal line is the mean over all country/time point groups. The graphs depicted in Figure 4 suggest that the mean of universalism increases over time in most countries, while it remains more or less stable in Portugal, Ireland, Finland, and Belgium.

\section{Summary and Conclusions}

In most published cross-national studies, metric and scalar measurement invariance is implicitly assumed without testing this assumption. This may lead to biased mean comparisons and biased comparisons of covariances and regression coefficients (Vandenberg and Lance, 2000; Jouha and Moustaki, 2013; Oberski, 2014). However, the traditional estimation procedures used in MGCFA to test for measurement invariance and the corresponding global fit measures, especially in the case of scalar invariance assessments, mostly lead to a rejection of the assumption of even partial invariance. This often results in a considerable reduction in the number of countries and/or time points whose means can be meaningfully compared.

In the current study we assessed the comparability of the universalism value in six rounds of the ESS between 2002 and 2012 across all ESS countries, with 90 country/time point

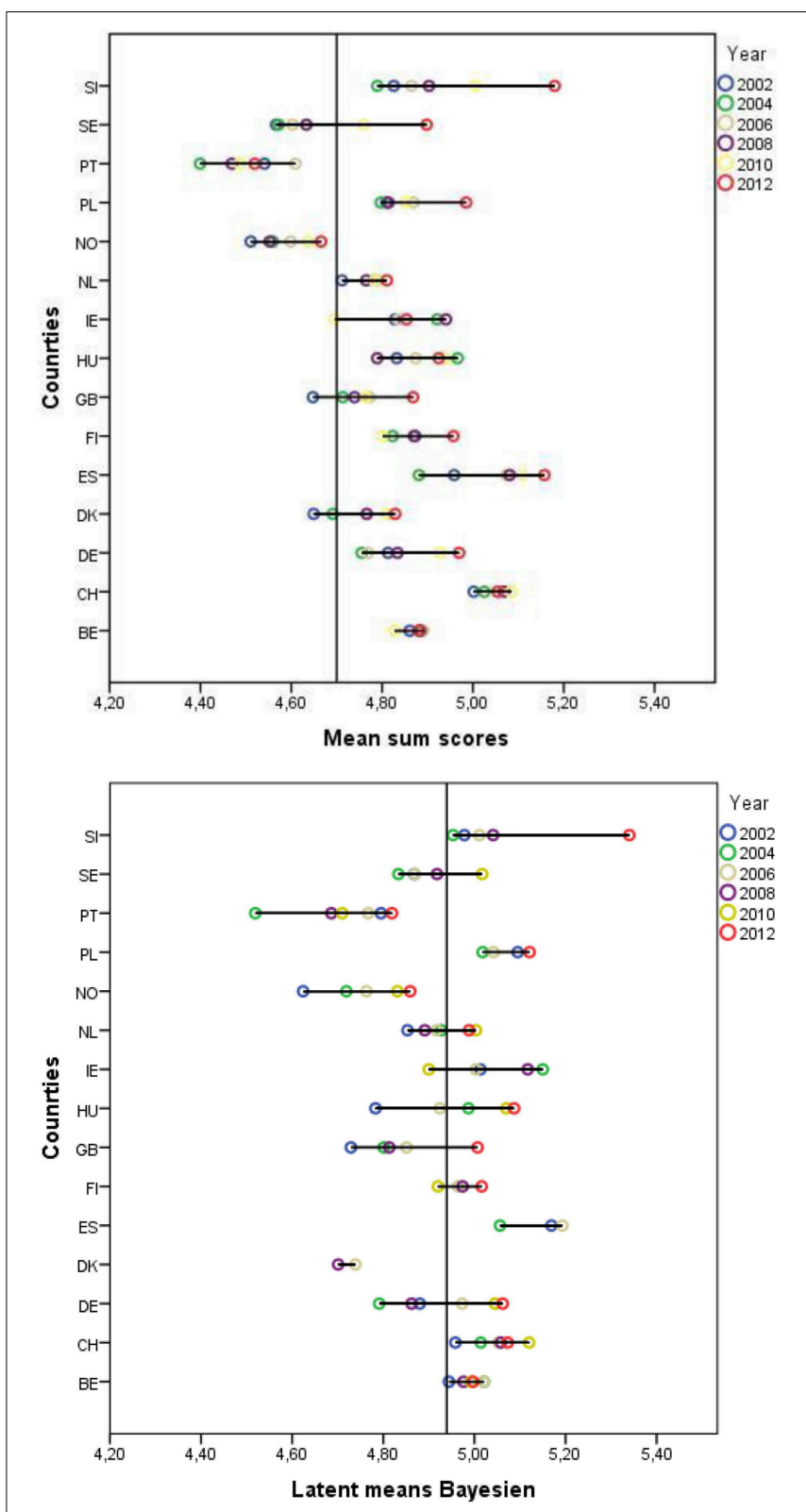

FIGURE 3 | Latent mean differences between 2002 and $2012^{21}$.

combinations in total. To the best of our knowledge, this is the first time in which so many groups are included in such a test. Using the traditional exact measurement invariance test procedure, metric invariance could be established across all country/time point combinations although partial scalar invariance could not, and we were required to drop almost two thirds of the countries/time points based on the reason that their mean scores on the scale might not be comparable.

\footnotetext{
${ }^{21}$ In the figure with the Bayesian latent means not all countries and time points are included. Countries/time points which are not included are Denmark 2000, 2002, 2010, 2012; Spain 2008, 2010, 2012; Finland 2000, 2002; United Kingdom 2010; Hungary 2008; Ireland 2012; Norway 2008; Poland 2008, 2010; Sweden 2012; Slovenia 2010.
} 

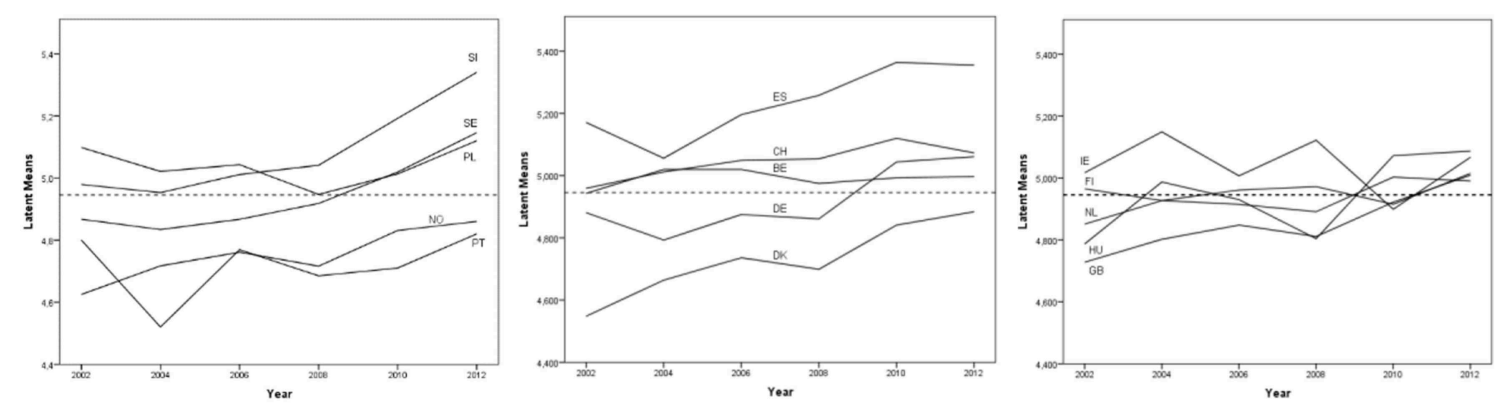

FIGURE 4 | Latent means over different time points and different countries ${ }^{22}$.

To solve this problem we applied the newly proposed approximate measurement invariance procedure. In these analyses only 17 country/time point combinations had to be excluded. We could demonstrate that the assumption of (approximate) scalar invariance was tenable using this alternative procedure on the remaining countries. As a consequence, the latent means of universalism could be legitimately compared across many more countries and time points.

Having said that, we believe that the traditional exact approach should always be applied as a first step in invariance testing. After all, it could well be the case that measurements are exactly invariant and it is not necessary to apply approximate (rather than exact) constraints. Using only the exact approach may circumvent not only using the (technically more challenging) approximate approach but a practical problem we encountered while analyzing the data applying the approximate approach as well: Using it for so many groups with large sample sizes led to a computation time of between 12 and $16 \mathrm{~h}$ ! However, where even partial exact measurement invariance does not hold, it would be useful to apply the approximate approach using Bayesian estimation (van de Schoot et al., 2013). This may be a relevant assessment especially in the case of comparisons of many groups such as in cross-national research with repeated cross-sections. As previous studies have demonstrated, in such cases it may be particularly difficult to establish full or partial (exact) scalar invariance.

It should be noted, however, that such a result in which so many country/time point combinations demonstrate approximate invariance may not necessarily be replicated with other data and other scales. Indeed, it could well be the case that both exact and approximate approaches fail to demonstrate cross-country and over time invariance. In other words, the approximate approach does not establish invariance where it is not given. It is, however, more liberal than the exact approach and may establish approximate invariance although the exact test fails to do so.

\footnotetext{
${ }^{22}$ Note that when certain time points were not available we extrapolated the data.
}

Future research may analyze various cross-national datasets with large samples to evaluate the approximate comparability of various scales and the practical usefulness of the approximate approach used here. In addition, it would be desirable if further simulation studies would be performed to evaluate which priors may be used in approximate invariance tests and which ppp values should be considered supportive for the assessed models. Such simulations could also explore how increasing the number of groups and the number of respondents in the groups may influence the results. This issue is particularly relevant because the number of groups (such as countries, cultural groups, language groups, etc.) in large data-generating programs such as the ESS, EVS, Eurobarometer, WVS, or the PISA study is continuously increasing. Furthermore, given that very often invariance cannot be established, it would be desirable if future studies would seek explanations for the absence of measurement invariance (see, e.g., Davidov et al., 2012, 2015). Finally, future research which includes a large number of groups may also apply other recent developments of testing for measurement invariance such as the alignment procedure (see, e.g., Muthén and Asparouhov, 2013) and examine the comparability of their findings to those of other more established approaches to test for invariance. Hopefully these methods and our empirical demonstration will encourage and support substantive researchers in their endeavor to conduct meaningful comparative research.

\section{Acknowledgments}

The authors would like to thank Lisa Trierweiler for the English proof of the manuscript. The work of the JC and ED was supported by the University Research Priority Program "Social Networks" of the University of Zürich.

\section{Supplementary Material}

The Supplementary Material for this article can be found online at: http://journal.frontiersin.org/article/10.3389/fpsyg. 2015.00733/abstract 


\section{References}

Beckers, T., Siegers, P., and Kuntz, A. (2012). Congruence and performance of value concepts in social research. Surv. Res. Method 6, 13-24.

Beierlein, C., Davidov, E., Schmidt, P., Schwartz, S., and Rammstedt, B. (2012). Testing the discriminant validity of Schwartz' Portrait Value Questionnaire items - a replication and extension of Knoppen and Saris (2009). Surv. Res. Method 6, 25-36.

Billiet, J., Maddens, B., and Beerten, R. (2003). National identity and attitude toward foreigners in a multinational state: a replication. Polit. Psychol. 24, 241-257. doi: 10.1111/0162-895X.00327

Bollen, K. A. (1989). Structural Equations with Latent Variables. New York, NY: Wiley. doi: 10.1002/9781118619179

Braun, M., Behr, D., and Kaczmirek, L. (2013). Assessing cross-national equivalence of measures of Xenophobia: evidence from probing in web surveys. Int. J. Public Opin. R. 25, 383-395. doi: 10.1093/ijpor/eds034

Brown, T. A. (2006). Confirmatory Factor Analysis for Applied Research. New York, NY: Guilford Press.

Byrne, B. M., Shavelson, R. J., and Muthén, B. O. (1989). Testing for the equivalence of factor covariance and mean structures-the issue of partial measurement invariance. Psychol. Bull. 105, 456-466. doi: 10.1037/0033-2909.105.3.456

Chen, F. F. (2007). Sensitivity of goodness of fit indexes to lack of measurement invariance. Struct. Equ. Modeling 14, 464-504. doi: 10.1080/10705510701301834

Cheung, G. W., and Rensvold, R. B. (2002). Evaluating goodness of fit indexes to lack of measurement invariance. Struct. Equ. Modeling 9, 233-255. doi: 10.1207/S15328007SEM0902 5

Cieciuch, J., Davidov, E., Schmidt, P., Algesheimer, R., and Schwartz, S. H. (2014a). Comparing results of an exact versus an approximate (Bayesian) measurement invariance test: a cross-country illustration with a scale to measure 19 human values. Front. Psychol. 5:982. doi: 10.3389/fpsyg.2014.00982

Cieciuch, J., Davidov, E., Vecchione, M., Beierlein, C., and Schwartz, S. H. (2014b). The cross-national invariance properties of a new scale to measure 19 basic human values a test across eight countries. J. Cross Cult. Psychol. 45, 764-776. doi: $10.1177 / 0022022114527348$

Datler, G., Jagodzinski, W., and Schmidt, P. (2013). Two theories on the test bench: internal and external validity of the theories of Ronald Inglehart and Shalom Schwartz. Soc. Sci. Res. 42, 906-925. doi: 10.1016/j.ssresearch.2012.12.009

Davidov, E. (2008). A cross-country and cross-time comparison of the human values measurements with the second round of the European Social Survey. Surv. Res. Method 2, 33-46.

Davidov, E. (2010). Testing for comparability of human values across countries and time with the third round of the European Social Survey. Int. J. Comp. Sociol. 51, 171-191. doi: 10.1177/0020715210363534

Davidov, E., Cieciuch, J., Schmidt, P., Meuleman, B., and Algesheimer, R. (2015). The comparability of measurements of attitudes toward immigration in the European Social Survey: exact versus approximate measurement equivalence. Public Opin. Qurterly 79, 244-266. doi: 10.1093/poq/nfv008

Davidov, E., Dülmer, H., Schlüter, E., Schmidt, P., and Meuleman, B. (2012). Using a multilevel structural equation modeling approach to explain crosscultural measurement noninvariance. J. Cross Cult. Psychol. 43, 558-575. doi: $10.1177 / 0022022112438397$

Davidov, E., Meuleman, B., Cieciuch, J., Schmidt, P., and Billiet, J. (2014a). Measurement equivalence in cross-national research. Annu. Rev. Sociol. 40, 55-75. doi: 10.1146/annurev-soc-071913-043137

Davidov, E., Meuleman, B., Schwartz, S. H., and Schmidt, P. (2014b). Individual values, cultural embeddedness, and anti-immigration sentiments: explaining differences in the effect of values on attitudes toward immigration across Europe. Kölner Z. Soz. Sozpsychol. 66, 263-285. doi: 10.1007/s11577-0140274-5

Davidov, E., Schmidt, P., and Schwartz, S. (2008). Bringing values back in. The adequacy of the European Social Survey to measure values in 20 countries. Public Opin. Q. 72, 420-445. doi: 10.1093/poq/nfn035

Davidov, E., and Siegers, P. (2010). "Comparing basic human values in East and West Germany", in Komparative Empirische Sozialforschung [Comprative Empirical Social Research], eds T. Beckers, K. Birkelbach, J. Hagenah, and U. Rosar (Wiesbaden: Verlag), 43-63. doi: 10.1007/978-3-531-92472-4_2
Gelman, A. (2003). A Bayesian formulation of explanatory data analysis and goodness of fit testing. Int. Stat. Rev. 71, 369-382. doi: 10.1111/j.17515823.2003.tb00203.x

Gelman, A. (2013). Two simple examples for understanding posterior p-values whose distributions are far from unform. Electron. J. Statist. 7, 2595-2602. doi: 10.1214/13-EJS854

Harkness, J. A., Braun, M., Edwards, B., Johnson, T. P., Lyberg, L., Mohler, P. P., (eds.) et al. (2010). Survey Methods in Multinational, Multiregional, and Multicultural Contexts. Hoboken, NJ: John Wiley \& Sons. doi: 10.1002/9780470609927

Hitlin, S., and Piliavin, J. A. (2004). Values: reviving a dormant concept. Annu. Rev. Sociol. 30, 359-393. doi: 10.1146/annurev.soc.30.012703.110640

Horn, J. L., and McArdle, J. J. (1992). A practical and theoretical guide to measurement invariance in aging research. Exp. Aging Res. 18, 117-144. doi: $10.1080 / 03610739208253916$

Inglehart, R. (1977). The Silent Revolution: Changing Values and Political Styles among Western Publics. Princeton, NJ: Princeton University Press.

Inglehart, R., and Welzel, C. (2005). Modernization, Cultural Change, and Democracy. The Human Development Sequence. Cambridge: Cambridge University Press.

Jöreskog, K. G. (1971). Simultaneous factor analysis in several populations. Psychometrika 36, 409-426. doi: 10.1007/BF02291366

Jöreskog, K. G. (1978). Structural analysis of covariance and correlation matrices. Psychometrika 43, 443-477. doi: 10.1007/BF02293808

Jöreskog, K. G. (1993). “Testing structural equation models," in Testing Structural Equation Models, eds K. A. Bollen and J. S. Long (Newbury Park, CA: Sage), 294-316.

Jouha, J., and Moustaki, I. (2013). Non-Equivalence of Measurement in Latent Variable Modelling of Multigroup Data: a Sensitivity Analysis. Available online at: http://ssrn.com/abstract=2332071

Jowell, R., Roberts, C., Fitzgerald, R., and Eva, G. (2007). Measuring Attitudes Cross-Nationally. Lessons from the European Social Survey. London: Sage.

Kaplan, D. (2014). Bayesian Statistics for the Social Sciences. New York, NY: Guilford Press.

Kass, R. E., and Raftery, A. E. (1995). Bayes factors. J. Am. Stat. Assoc. 90, 773-795. doi: 10.1080/01621459.1995.10476572

Kruschke, J. K., Aguinis, H., and Joo, H. (2012). The time has come! Bayesian methods for data analysis in the organizational sciences. Organ. Res. Methods 15, 722-752. doi: 10.1177/1094428112457829

Kuntz, A., Davidov, E., Schwartz, S. H., and Schmidt, P. (2015). Human values, legal regulation and approval of homosexuality in Europe: a cross-country comparison. Eur. J. Soc. Psychol. 45, 120-134. doi: 10.1002/ejsp.2068

Latcheva, R. (2011). Cognitive interviewing and factor-analytic techniques: a mixed method approach to validity of survey items measuring national identity. Qual. Quant. 45, 1175-1199. doi: 10.1007/s11135-009-9285-0

Levy, R. (2011). Bayesian data-model fit assessment for structural equation modeling. Struct. Equ. Modeling 18, 663-685. doi: 10.1080/10705511.2011.607723

Levy, R., and Choi, J. (2013). "Bayesian structural equation modeling," in Structural Equation Modeling. A Second Course, 2nd Edn., eds G. R. Hancock and R. O. Mueller (Charlottesville, NC: Information Age Publishing), 563-624.

Little, T. D., Slegers, D. W., and Card, N. A. (2006). A non-arbitrary method of identifying and scaling latent variables in SEM and MACS models. Struct. Equ. Modeling 13, 59-72. doi: 10.1207/s15328007sem1301_3

Meade, A. W., Johnson, E. C., and Braddy, P. W. (2008). Power and sensitivity of alternative fit indices in tests of measurement invariance. J. Appl. Psychol. 93, 568-592. doi: 10.1037/0021-9010.93.3.568

Meredith, W. (1993). Measurement invariance, factor analysis and factorial invariance. Psychometrika 58, 525-543. doi: 10.1007/BF02294825

Meuleman, B. (2012). "When are intercept differences substantively relevant in measurement invariance testing," in Methods, Theories, and Empirical Applications in the Social Sciences: Festschrift for Peter Schmidt, eds S. Salzborn, E. Davidov, and J. Reinecke (Wiesbaden: Springer), 97-104.

Meuleman, B., Davidov, E., Schmidt, P., and Billiet, J. (2012). "Social location and value priorities: a European-wide comparison of the relation between social-structural variables and human values," in Society and Democracy in Europe, eds O. Gabriel and S. I. Keil (London: Routledge), 45-67. 
Millsap, R. E. (2011). Statistical Approaches to Measurement Invariance. New York, NY: Routledge.

Muthén, B. O., and Asparouhov, T. (2012). Bayesian structural equation modeling: a more flexible representation of substantive theory. Psychol. Methods 17, 313-335. doi: 10.1037/a0026802

Muthén, B. O., and Asparouhov, T. (2013). BSEM Measurement Invariance Analysis. Mplus Web Notes: No. 17. Available online at: http://www.statmodel. com

Muthén, L., and Muthén, B. O. (1998-2014). Mplus User's Guide, 7th Edn. Los Angeles, CA: Muthén and Muthén.

Oberski, D. L. (2014). Evaluating sensitivity of parameters of interest to measurement invariance in latent variable models. Polit. Anal. 22, 45-60. doi: $10.1093 / \mathrm{pan} / \mathrm{mpt} 014$

Oberski, D. L., Weber, W., and Revilla, M. (2012). "The effect of individual characteristics on reports of socially desirable attitudes towards immigration," in Methods, Theories, and Empirical Applications in the Social Sciences: Festschrift for Peter Schmidt, eds S. Salzborn, E. Davidov, and J. Reinecke (Wiesbaden: Springer), 151-158.

Opp, K.-D. (2011). Modeling micro macro relationships: problems and solutions. J. Math. Sociol. 35, 209-234. doi: 10.1080/0022250X.2010. 532257

Piurko, Y., Schwartz, S. H., and Davidov, E. (2011). Basic personal values and the meaning of left-right political orientations in 20 countries. Polit. Psychol. 32, 537-561. doi: 10.1111/j.1467-9221.2011.00828.x

Popper, K. R. (2005). The Logic of Scientific Discovery. London: Routledge.

Saris, W., Knoppen, D., and Schwartz, S. (2013). Operationalizing the theory of human values: balancing homogeneity of reflective items and theoretical coverage. Surv. Res. Method 7, 29-44.

Saris, W., Satorra, A., and van der Veld, W. M. (2009). Testing structural equation models or detection of misspecifications? Struct. Equ. Modeling 16, 561-582. doi: 10.1080/10705510903203433

Sarrasin, O., Green, E. G. T., Berchtold, A., and Davidov, E. (2012). Measurement equivalence across subnational groups: an analysis of the conception of nationhood in Switzerland. Int. J. Public Opin. R. 25, 522-534. doi: 10.1093/ijpor/eds033

Schafer, J., and Graham, J. (2002). Missing data: our view of the state of the art. Psychol. Methods 7, 147-177. doi: 10.1037/1082-989X.7.2.147

Schwartz, S. H. (2003). "A proposal for measuring value orientations across nations," in Questionnaire Development Package of the European Social Survey, Chapter 7. Available online at: www.europeansocialsurvey.org
Schwartz, S. H., Cieciuch, J., Vecchione, M., Davidov, E., Fischer, R., Beierlein, C., et al. (2012). Refining the theory of basic individual values. J. Pers. Soc. Psychol. 103, 663-688. doi: 10.1037/a0029393

Schwarz, G. (1978). Estimating the dimension of a model. Ann. Statist. 6, 461-464. doi: 10.1214/aos/1176344136

Spiegelhalter, D. J., Best, N. G., Carlin, B. P., and van der Linde, A. (2002). Bayesian measure of model complexity and fit. J. R. Statist. Soc. B. 64, 583-639. doi: $10.1111 / 1467-9868.00353$

Steenkamp, J.-B. E. M., and Baumgartner, H. (1998). Assessing measurement invariance in cross-national consumer research. J. Consum. Res. 25, 78-90. doi: $10.1086 / 209528$

Steinmetz, H. (2013). Analyzing observed composite differences across groups: is partial measurement invariance enough? Methodology 9, 1-12. doi: 10.1027/1614-2241/a000049

Thompson, M. S., and Green, S. B. (2013). "Evaluating between group differences in latent variable means," in Structural Equation Modeling. A Second Course, 2nd Edn., eds G. R. Hancock and R. O. Mueller (Charlottesville, VA: Information Age Publishing), 163-218.

van de Schoot, R., Kluytmans, A., Tummers, L., Lugtig, P., Hox, J., and Muthén, B. O. (2013). Facing off with Scylla and Charybdis: a comparison of scalar, partial, and the novel possibility of approximate measurement invariance. Front. Psychol. 4:770. doi: 10.3389/fpsyg.2013.00770

van de Schoot, R., Lugtig, P., and Hox, J. (2012). A checklist for testing measurement invariance. Eur. J. Dev. Psychol. 9, 486-492. doi: 10.1080/ 17405629.2012.686740

Vandenberg, R. J., and Lance, C. E. (2000). A review and synthesis of the measurement invariance literature: suggestions, practices, and recommendations for organizational research. Organ. Res. Methods 3, 4-70. doi: $10.1177 / 109442810031002$

Conflict of Interest Statement: The authors declare that the research was conducted in the absence of any commercial or financial relationships that could be construed as a potential conflict of interest.

Copyright (c) 2015 Zercher, Schmidt, Cieciuch and Davidov. This is an open-access article distributed under the terms of the Creative Commons Attribution License (CC $B Y)$. The use, distribution or reproduction in other forums is permitted, provided the original author(s) or licensor are credited and that the original publication in this journal is cited, in accordance with accepted academic practice. No use, distribution or reproduction is permitted which does not comply with these terms. 